\title{
STABILIZATION OF NONHOLONOMIC MOBILE ROBOTS BY A GA-BASED FUZZY SLIDING MODE CONTROL
}

\author{
Wei-Ming Lee \\ Graduate Student \\ Han-Pang Huang \\ Professor \\ Department of Mechanical Engineering, \\ National Taiwan University, Taipei, Taiwan, 10764 R.O.C.
}

\begin{abstract}
In this study, fuzzy logic control principle and sliding mode control theory are combined to stabilize a mobile robot under nonholonomic kinematic constraints. In order to improve the tracking performance, the scaling factors of FLC are searched by genetic algorithms (GAs). The simulation results show that all three control approaches, SMC (sliding mode control), FSMC (fuzzy-SMC) and GA-FSMC (GA-based FSMC) can stabilize the mobile robot to the origin with $\phi=0$. However, the GA-FSMC provides a systematic way to search the scaling factors, and thus improves the transient performance of the system response and reduces the path length for navigation.
\end{abstract}

\section{Introduction}

Fuzzy logic controllers (FLC) [2] have been widely and successfully applied to a variety of control problems. Essentially, the FLC provides an algorithm to convert a linguistic control strategy described by IF-THEN rules into an automatic control strategy. Fuzzy logic is much closer to human decision making than traditional binary logic. Thus, FLC provides a new design method for those problems which were not or uneasily synthesized in the past.

There has been a great deal of research recently on the problem of stabilizing nonholonomic systems $[5,6]$ which are of importance for numerous practical applications, such as mobile robots. Brockett's theorem [7] proved that for any nonholonomic system the equilibrium point of the system cannot be asymptotically stabilized by any smooth (or even continuous) static time-invariant state feedback controller. Several discontinuous time-invariant feedback controllers $[5,6]$ were developed. Although the sliding mode control [6] can stabilize the nonholonomic system, the transient performance of the system is not good enough. In this study, a fuzzy sliding mode control with genetic algorithms (GA-FSMC) is proposed for the navigation of a mobile robot under nonholonomic kinematic constraints. The genetic algorithm [3] is used to globally search for the near-optimal scaling factor of the fuzzy controller. The fitness value is evaluated in terms of system time response characteristics, such as rise time, maximum overshoot and settling time weighted by means of Guassian membership functions. It will shown that GAFSMC improves the performance of tracking the direction of velocity and shortens the traversal path length. 
A typical example of a wheeled mobile robot is shown Fig. 1. The kinematics for the robot can be described by the following equations

$$
\begin{aligned}
& \dot{\mathrm{x}}=\frac{1}{2}\left(\mathrm{v}_{\mathrm{R}}+\mathrm{v}_{\mathrm{L}}\right) \cos \phi \\
& \dot{\mathrm{y}}=\frac{1}{2}\left(\mathrm{v}_{\mathrm{R}}+\mathrm{v}_{\mathrm{L}}\right) \sin \phi \\
& \dot{\phi}=\frac{1}{2 \mathrm{D}}\left(\mathrm{v}_{\mathrm{R}}-\mathrm{v}_{\mathrm{L}}\right)
\end{aligned}
$$

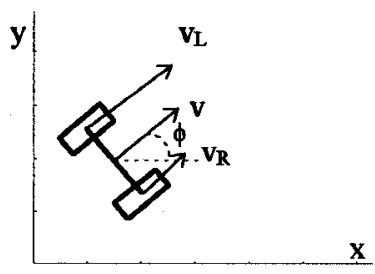

Fig 1 A wheeled mobile robot

For control analysis and design, kinematic equations (1) are transformed into the control input decoupled form by the substitutions $v=0.5\left(v_{R}+v_{L}\right), \omega=0.5\left(v_{R}-v_{L}\right)$ and then the kinematic equations (1) can be rewritten as

$$
\begin{aligned}
& \dot{\mathrm{x}}=v \cos \phi \\
& \dot{\mathrm{y}}=\mathrm{v} \sin \phi \\
& \dot{\phi}=\omega
\end{aligned}
$$

The transformed control inputs are the steering angular velocity of the robot $\omega$ and the translational velocity of the mobile robot $\mathrm{v}$. Note that the direction of $\mathrm{v}$ is orthogonal to the axis of the rear wheels due to the nonholonomic constraints. For navigating the wheeled set to the origin of working space, a Lyapunov function $\mathrm{V}(\mathrm{x}, \mathrm{y})[6]$ can be designed so that the associated gradient $\mathrm{E}(\mathrm{x}, \mathrm{y})=-\operatorname{grad}(\mathrm{V})$ determines the desired orientation of the robot. The corresponding orientation equation is described as

$$
\frac{d x}{d y}=\frac{\partial E(x, y) / \partial x}{\partial E(x, y) / \partial y}=\frac{E_{x}(x, y)}{E_{y}(x, y)}
$$

control method

In this section, three control approaches, SMC, FSMC and GA-FSMC, for the nonholonomic dynamic system are examined respectively:

(1) Sliding mode control [6]

By taking the gradient lines $E=-\operatorname{grad}(V)$ of the Lyapunov function described in the previous section as the sliding manifold, the orientation of the robot is driven to the gradient field to achieve $\phi / / \phi_{\mathrm{R}}\left(\phi_{\mathrm{R}}=\operatorname{atan}(\mathrm{Ex} / \mathrm{Ey})\right.$ ) in the reaching mode and restricted invariantly to the gradient field in the sliding mode. Let $s=\Delta \phi=\phi-\phi_{\mathrm{R}}$. By differentiating $\Delta \phi$ along the gradient fields $\mathrm{E}(\mathrm{x}, \mathrm{y})$ and using Eq.(2), we have

$$
\begin{aligned}
\dot{s}=\frac{d}{d t}(\Delta \phi) & =\omega-\mathrm{F}(\mathrm{x}, \mathrm{y}, \phi) \mathrm{v}(\mathrm{t}) \\
\mathrm{F}(\mathrm{x}, \mathrm{y}, \phi) & =\frac{\mathrm{E}_{\mathrm{x}} \mathrm{E}_{\mathrm{y}, \mathrm{x}}-\mathrm{E}_{\mathrm{y}} \mathrm{E}_{\mathrm{x}, \mathrm{y}}}{\|\mathrm{E}\|^{2}} \cos \phi+\frac{\mathrm{E}_{\mathrm{x}} \mathrm{E}_{\mathrm{y}, \mathrm{y}}-\mathrm{E}_{\mathrm{y}} \mathrm{E}_{\mathrm{x}, \mathrm{y}}}{\|\mathrm{E}\|^{2}} \sin \phi
\end{aligned}
$$

The control law is

$$
\omega=\mathrm{Fv}(\mathrm{t})-\xi|\Delta \phi|^{\frac{1}{2}} \operatorname{sign}(\Delta \phi)
$$


where $\xi>0$ is a finite scalar constant. Substituting (5) into (4), it yields

$$
(\Delta \phi) \frac{\mathrm{d}}{\mathrm{dt}}(\Delta \phi)=-(\Delta \phi) \xi|\Delta \phi|^{\frac{1}{2}} \operatorname{sign}(\Delta \phi) \leq 0
$$

Eq.(6) satisfies the sliding condition so that the orientation of the mobile robot can be driven into the sliding manifold in the reaching mode.

(2) Fuzzy-sliding mode control (FSMC) [1,4]

The PI-like FSMC is shown in Fig. 2, where $S, \dot{S}$ and $d \Omega$ are fuzzy variables. The universes of discourse of these fuzzy variables are ranged from -3 to 3 . Each fuzzy variable is quantified into seven term sets : NB-Negative Big, NM-Negative Medium, NS-Negative Small, ZE-Zero, PS-Positive Small, PM-Positive Medium, PB-Positive Big. The triangular membership functions for each fuzzy term set are shown in Fig 3. The crisp values s, $\dot{s}$ and $d \omega$ of FSMC are scaled by Gs, Gcs and $\mathrm{G} \omega$, respectively. Based on equations (5)(6), the resultant control rules are shown in Table 1. Some of the rules can be explained as follows.

IF $\mathrm{S}$ is NB and $\dot{\mathrm{S}}$ is NB, THEN $\mathrm{d} \Omega$ is $\mathrm{PB}$

This control rule expresses that if the states $S$ and $\dot{S}$ are both negative big, which means that the state trajectories are leaving away the sliding manifold in the direction of decreasing the value of $s$, then the change of control do should be positive big so that the state $\dot{s}$ would be increasing to draw the state $s$ back to zero quickly.

IF $S$ is NB and $\dot{S}$ is PB, THEN $d \Omega$ is $Z E$

This control rule implies that if the states $S$ is negative big and the states $\dot{S}$ is positive big, which means that the state trajectories are approaching the sliding manifold properly, then the control input $\omega$ need not be tuned.

\section{(3) GA-based FSMC}

In this approach, the scaling factors of FLC will be globally searched for nearoptimal values. The string (chromosome) representing the scaling factors is integer-based rather than binary-based. The value of each gene for chromosome ranges from 0 to $n r$, an integer value which stands for the resolution of the discretized scaling factors. The discretized scaling factors can be decoded as

$$
\mathrm{sc}_{\mathrm{i}}=\left(\max _{\mathrm{i}}-\min _{\mathrm{i}}\right) \times \frac{\text { gene }}{\mathrm{nr}}+\min _{\mathrm{i}}
$$

The fitness value assigned to each string is evaluated according to the system time response characteristics, e.g. rise time (rt), maximum overshoot (ov) and settling time (st). These factors are fuzzified with Guassian membership function so that the time response can be flexibly evaluated. The corresponding weights of three time response indexes are determined by the $\delta_{i}$ of $\mathrm{G}\left(\mathrm{x}, \delta_{\mathrm{i}}\right)=\exp \left(\frac{-\mathrm{x}^{2}}{\delta_{\mathrm{i}}^{2}}\right)$.

There are three basic genetic operators: reproduction, crossover and mutation. They can be briefly described as follows.

(1) Reproduction 
Reproduction is based on the principle of survival of the better fitness. Those strings with large fitness will have a large number of copies in the new generation.

\section{(2) Crossover}

Crossover provides a mechanism for chromosome to mix each other through a random process. First, two strings with high fitness are selected from the mating pool produced by reproduction. Second, a position called cross-site along the two strings is selected at random. Finally, the exchange of the two selected strings takes place in the cross-site . For example, $n r=20$,

\begin{tabular}{|c|c|c|c|c|c|c|c|c|c|c|c|c|c|c|c|}
\hline Parent & & & cros & s-sit & & & Of & prin & & & & & & & \\
\hline$\# 1:$ & 4 & 12 & 7 & 3 & 9 & 5 & $\Rightarrow$ & \#1: & 4 & 0 & 12 & 7 & 2 & 15 & 19 \\
\hline \#2: & $\begin{array}{ll}6 & 17\end{array}$ & 2 & 11 & 2 & 15 & 19 & $\Rightarrow$ & \#2: & 6 & 17 & 2 & 11 & 3 & 0 & - \\
\hline
\end{tabular}

\section{(3) Mutation}

The mutation operator provides a method to improve the ability of the nearoptimal search. Mutation is a complement operation at a position of string via less mutation probability as follows.

$$
\begin{aligned}
& \text { mutation } \\
& \begin{array}{lllllll}
4 & 0 & : 12: & 7 & 2 & 15 & 19
\end{array} \\
& \begin{array}{ll:lllll}
4 & 0 & 12 & 7 & 2 & 15 & 19 \\
4 & 0 & 7 & 7 & 2 & 15 & 19
\end{array}
\end{aligned}
$$

In order to enhance the convergence of GA, when the maximum value of the fitness among the population is non-increasing, the rate of mutation will be increased until the maximum fitness value will be increasing again.

\section{Simulation}

In this section, a specific example of a Lyapunov function for navigating the wheeled mobile robot to the origin with the orientation $\phi=0$ will be examined. A quadratic Lyapunov function is defined as [6]

$$
V(x, y)=\frac{1}{2}\left(\frac{x^{2}}{a}+y^{2}\right)
$$

The following two cases $\left(x_{0}=0.1 y_{0}=4.0 \phi_{0}=\pi\right)$ are simulated:

case 1 - no disturbance

case 2 -- a translational disturbance 0.2 (normal to the trajectory), a rotational disturbance $\Delta \phi=1(\mathrm{rad})$

The SMC, FSMC and GA-FSMC will be compared in terms of these two cases.

(1) Sliding mode control [6]

The control law for orienting the mobile robot longitudinal axis co-linear to the gradient line is obtained by substituting (8) into (5)

$$
\omega=\mathrm{a} \frac{\mathrm{x} \sin \phi-\mathrm{y} \cos \phi}{\mathrm{x}^{2}+\mathrm{a}^{2} \mathrm{y}^{2}} \mathrm{v}-\xi|\Delta \phi|^{\frac{1}{2}} \operatorname{sign}(\Delta \phi), \quad \mathrm{a}=2.0, \xi=8.5
$$

\section{(2) FSMC [1]}

The inference rules in Table 1 and the membership functions in Fig. 3 are the same for all cases. The scaling factors are determined by trial and error as

$$
\text { for } 0<\mathrm{s}<0.2 \quad \mathrm{Gs}=5.0 \quad \mathrm{G} c \mathrm{~s}=10.0 \quad \mathrm{G} \omega=13.0|\mathrm{~s}|^{0.8}
$$




$$
\text { for } 0.2<\mathrm{s} \quad \mathrm{Gs}=10.0 \quad \mathrm{Gcs}=5.0 \quad \mathrm{G} \omega=3.0|\mathrm{~s}|^{0.8}
$$

(3) GA-FSMC

Instead of consuming the time to determine the scaling factors of FSMC, GA itself can be used to adjust the scaling factors in the near-optimal way. The scaling factors in two regions are determined as

$$
\begin{array}{llll}
\text { for } 0<\mathrm{s}<0.26 & \mathrm{Gs}=2.2 & \mathrm{G} c \mathrm{~s}=72.1 & \mathrm{G} \omega=15.0|\mathrm{~s}|^{0.8} \\
\text { for } 0.26<\mathrm{s} & \mathrm{Gs}=4.9 & \mathrm{G} c \mathrm{~s}=5.7 & \mathrm{G} \omega=5.2|\mathrm{~s}|^{0.8}
\end{array}
$$

In this study, the fitness value is determined so that the maximum overshoot is suppressed. The fitness value is $G(t, 1.5) \times G(o v, 0.5) \times G(s t, 3.0)$. The simulation results of these three controllers are shown in Fig. 4, Fig. 5, where, for comparing the tracking performance, Fig. $4 \mathrm{~d}$ and Fig .5d are zoomed out from Fig. $4 \mathrm{c}$, Fig. 5c, respectively.

\section{Conclusion}

Several conclusions can be drawn from the simulation results as follows:

(1) All three control approaches can stabilize the mobile robot to the origin with the desired orientation $\phi=0$.

(2) From the viewpoint of design and tracking performance, the fuzzy sliding mode control approach is easier and better than the original sliding mode control, but the tuning of the scaling factors is time-consuming.

(3) In GA-FSMC approach, the genetic algorithm provides a systematic procedure to search the scaling factors, and thus improves the transient performance of the system response and reduces the path for navigation.

\section{References}

[1] C.C. Kung and C.C. Lao, "Fuzzy-Sliding Mode Control of Non-Linear System," Automatic Control Conference, R.O.C., pp.259-264, 1994.

[2] C.C. Lee, "Fuzzy Logic in Cntrol Systems: Fuzzy Logic Controller-part I II," IEEE Trans. on SMC, vol. 20, no. 2, pp.404-435, 1990.

[3] D.E. Goldberg, Genetic Algorithms in Search, Optimization and Machine Learning. Reading, MA: Addison-Wesley, 1989.

[4] G.C. Hwang and S.C. Lin, "A stability Approach to Fuzzy Control Design for Nonlinear Systems," Fuzzy Sets and Systems, vol. 48, pp.279-287, 1992.

[5] I. Kolmanovsky and N.H. McClamroch, "Developments in Nonholonomic control problems," IEEE Control Systems, pp.20-36, 1995.

[6] J. Guldner and V.I. Utkin, "Stabilization of Nonholonomic Mobile Robots using Lyapunov Functions for Navigation and Sliding Mode Control," Proceedings of the 33rd IEEE CDC, pp.2967-2972, 1994.

[7] R.W. Brockett, "Asymptotic Stability and Feedback Stabilization," R.S. Millmann (eds.), Differential Geometric Control Theory, Birkhauser, Boston, MA, pp.181-191, 1983. 
[8] V.I. Utkin, Sliding Modes in Control and Optimization, Springer-Verlag, Berlin, 1992.
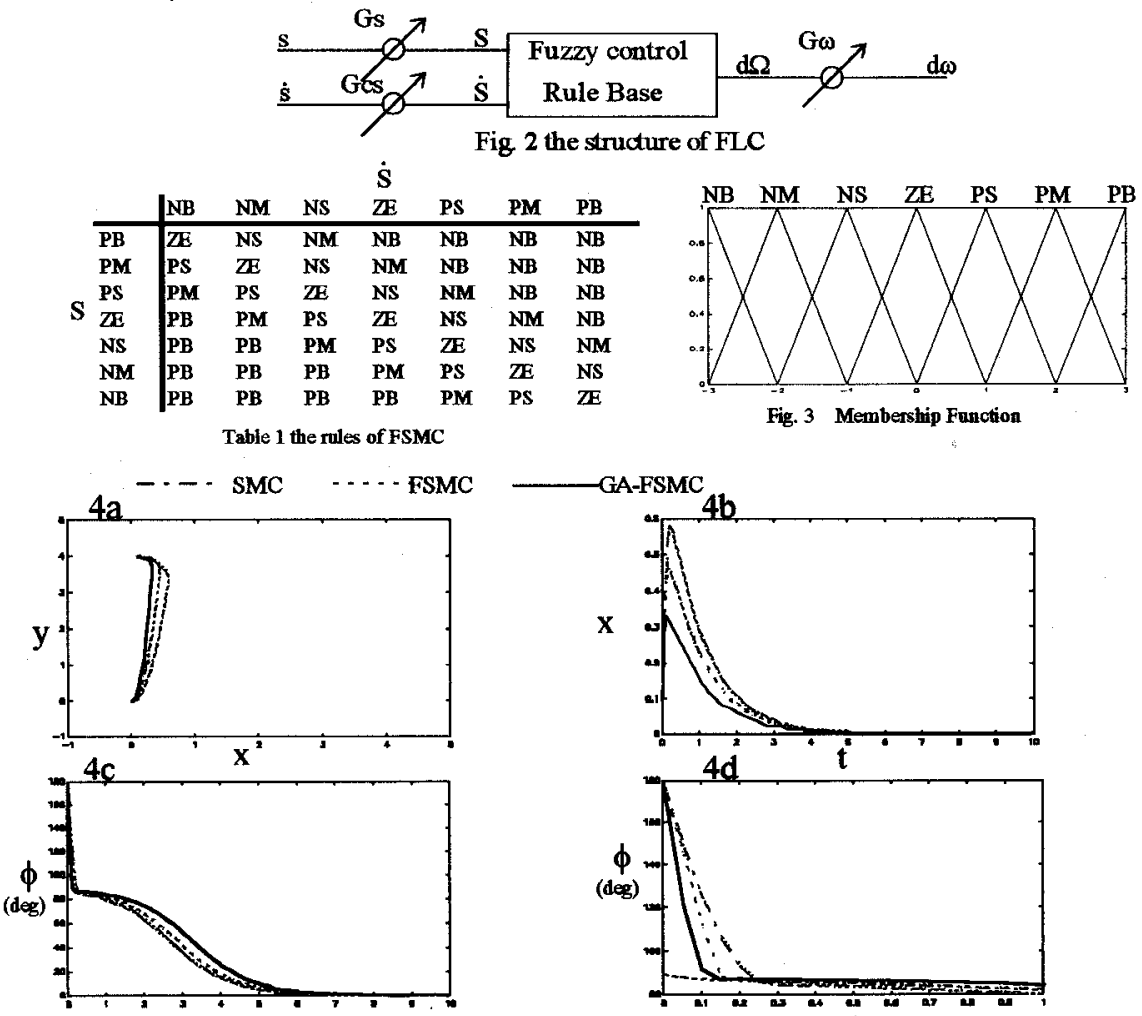

$t \quad$ Fig. 4 the simulation results for case $1 t$
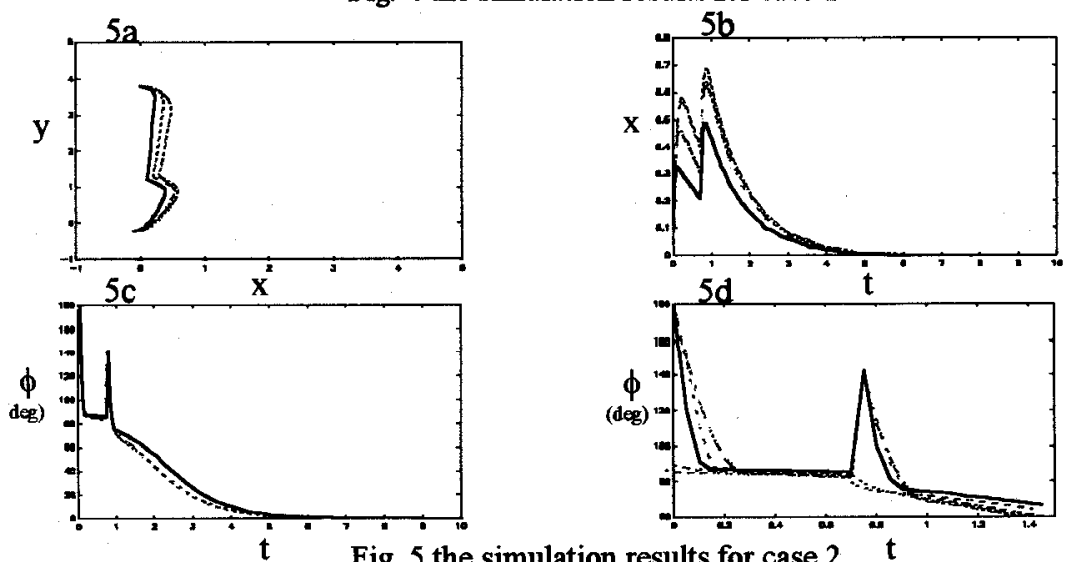

Fig. 5 the simulation results for case 2 